\title{
Prevalencia de leptospirosis en humanos en la zona urbana del municipio de Puerto Libertador, Córdoba, Colombia
}

\author{
Heidy Rodríguez Barreto ${ }^{12}$, Carlos Lozano Montoya ${ }^{2}$, \\ Carlos Bedoya Sánchez ${ }^{2}$ \& Leonardo Grondona Paternina ${ }^{2}$ \\ 'heidy.rodriguez@unad.edu.co \\ ${ }^{2}$ Escuela de Ciencias Agrícolas, Pecuarias y del Medio Ambiente. \\ Universidad Nacional Abierta y a Distancia
}

Resumen.- Es este estudio buscamos determinar la prevalencia de leptospirosis en humanos en situaciones de desplazamiento en la zona urbana del Municipio de Puerto Libertador, en la región Caribe de Colombia. Realizamos un estudio epidemiológico de corte transversal en el que tomamos muestras de suero e información general (ocupación, exposición a fuentes contaminadas como agua o animales) de 78 individuos. Los sueros fueron analizados mediante la prueba de microaglutinación. (MAT) con seis serovares de Leptospira. Encontramos anticuerpos antileptospirales en el $67.9 \%$ de las personas, siendo más frecuente en mayores de 20 años. De los seis serovares evaluados, los títulos de anticuerpos fueron altos especialmente para L. icterohaemorragiae. La alta prevalencia del serovar encontrado en humanos en la zona urbana del Municipio de Puerto Libertador sugiere la transmisión frecuente de leptospiras probablemente por la presencia de ratas o ratones en las viviendas, los aljibes al aire libre y canales de aguas lentas.

Palabras clave: Leptospira, leptospirosis, microaglutinación, serovar, zoonosis

\begin{abstract}
The objective of this research was to determine the prevalence of leptospirosis in humans in the urban area in Puerto Libertador, Cordoba, in the Caribean region of Colombia. In august 2007, we used a cross-sectional epidemiological study. We included serum samples from 78 individuals and general information (occupation, exposure to contaminated sources such as water or animals). We analyzed those samples through microscopic agglutination test (MAT) with six serovars of Leptospira. Anti-Leptospira antibodies were found in $67.9 \%$ of the sample, in patients older than 20 years, with no significant differences in the sex variable. Among six serovars tested, the antibody titers were high, especially L. icterohaemorragiae. The high prevalence of serovars in humans in the urban area in Puerto Libertador suggests frequent transmission of leptospires, whish is relatedto the presence of rats or mice in homes, outdoors and cisterns of slack water channels.
\end{abstract}

Key words: Leptospira, leptospirosis, microglutination, serovar, zoonoses

\section{Introducción}

La leptospirosis es una enfermedad común al hombre y algunos animales, que es causada por cualquiera de los microorganismos del género Leptospira. Esta es la zoonosis contemporánea más difundida en el mundo, excepto en las regiones polares que son las únicas zonas libres del microorganismo. Colombia, por su situación geográfica, no escapa de esta afección, que se presenta especialmente en las zonas predominantemente agropecuarias. Es una enfermedad que puede ser causada por más de 180 serovares de Leptospira interrogans, que fue descubierta por Weil en 1886, quien describió el cuadro clínico clásico (Torales 2002, Sandow \& Ramírez 2005).

Los Leptospira son microorganismos Gramnegativos, aerobios obligados, móviles, delgados, flexibles y enrollados en forma helicoidal.. $\mathrm{Su}$ nombre se deriva de la palabra lepto que 
significa espiral delgada o fina. Miden de 6 a $20 \mu \mathrm{m}$ de longitud y 0.1 a $0.5 \mu \mathrm{m}$ de diámetro. La enfermedad que produce afecta o está relacionada con animales domésticos y silvestres, como perros, gatos, bovinos, caprinos, porcinos, caballos, roedores, mamíferos marinos, entre otros (Torales 2002) y puede afectar también a los humanos.

La bacteria se transmite fundamentalmente a través de la orina de animales infectados, aunque puede encontrarse también en otros fluidos corporales. Por lo general, os animales infectados, como roedores y otros animales silvestres, no presentan síntomas. La Leptospi$r a$ se disemina rápidamente en todos los tejidos, excepto el cerebro, los ojos y los riñones, donde sobrevive y se multiplica para luego eliminarse a través de la orina. La bacteria permanece en el huésped por semanas o meses y en el caso de los roedores puede reproducirse y ser eliminada durante toda la vida (Nájera et al.2005).

En Colombia la leptospirosis no es una enfermedad de notificación obligatoria y se desconocen tanto su prevalencia como su impacto económico, aunque se han descrito casos aislados en humanos y brotes en varios municipios a lo largo del país. Entre 2000 y 2003 se conocieron estudios de prevalencia de leptospirosis, uno en personas sintomáticas en la ciudad de Cali, Valle del Cauca, y el otro en trabajadores agrícolas en el municipio de Don Matías, Antioquia,, con tasas de ataque de 6.4 y $22.8 \%$, respectivamente. En el departamento de Córdoba, región ganadera con clima tropical propicio para el desarrollo de esta zoonosis, se cuenta con estudios de prevalencia de leptospirosis en porcinos, cuya tasa de infección oscila entre 30 y $50 \%$ y un estudio en humanos con una prevalencia de $13.1 \%$ (Ochoa et al. 2000).

El objetivo de este estudio fue determinar la prevalencia de leptospirosis en humanos en situaciones de desplazamiento, en la zona urbana del Municipio de Puerto Libertador, departamento de Córdoba, en la región Caribe de Colombia, mediante la implementación de la prueba de microalgutinación (MAT).

\section{Métodos}

En agosto de 2007 realizamos un estudio epidemiológico de corte transversal de preva- lencia de leptospirosis en los barrios Ramón Rubio y Siete de Septiembre, en el Municipio de Puerto Libertador, Córdoba, donde hay una población aproximada de 780 personas en situación de desplazamiento. Para calcular la prevalencia de infección por Leptospira estimamos un tamaño de muestra del $10 \%$ del total de la población ( 78 personas). A las personas que hicieron parte de la muestra, que fueron seleccionadas al azar mediante la generación de números aleatorios con una calculadora, les explicamos claramente los objetivos, procedimientos, posibles riesgos y beneficios, y les solicitamos una autorización por escrito mediante una firma de carta de consentimiento informado. Adicionalmente tomamos datos epidemiológicos como edad, sexo, localización, así como la ocupación y factores potencialmente asociados con leptospirosis, como fuentes de agua utilizadas o contacto con animales domésticos ( $v, g r$. perros, cerdos, etc.) o peridomésticos ( $v$.gr. roedores).

Procesamiento de las muestras.- Por medio de venopunción en la fosa antecubital del brazo, obtuvimos $5 \mathrm{ml}$ de sangre de cada paciente. Las muestras fueron recolectadas en tubos de Vaicutainer ${ }^{\circledR}$ sin anticoagulante y el suero fue congelado a menos $20^{\circ} \mathrm{C}$. Para el análisis e interpretación de las muestras realizamos una prueba MAT, con diluciones de 1:100, 1:200 y 1:400 del suero con solución salina amortiguada con fosfatos (SAF). Como control positivo usamos un suero con títulos conocidos de acuerdo al serovar en estudio. Diluimos este control igual que las muestras y utilizamos solución salina amortiguada con fosfatos (SAF) como control de antígenos. Posteriormente mezclamos el suero con el SAF durante 20 minutos a temperatura ambiente; en microplacas rígidas pusimos $50 \mu \mathrm{l}$ de la dilución del suero y posteriormente los controles. Adicionamos $50 \mu \mathrm{l}$ del antígeno previamente preparado y mezclamos el suero con los respectivos serovares a utilizar (L. pomona, L. canicola, L. bratislava, L. grippotyphosa, L. icterohaemorragiae, $L$. hardjo). La incubación duró dos horas a $30^{\circ} \mathrm{C}$ y posteriormente observamos en un microscopio de campo oscuro con objetivo de $10 \mathrm{X}$ colocando una gota en una lámina portaobjetos. La prueba se interpretó como positiva cuando había una aglutinación igual o mayor al 50\% de las leptospiras con cualquiera de los serovares, en las diferentes 
Tabla 1. Niveles de titulación de los diferentes serovares de Leptospira en humanos en la zona urbana del municipio de puerto libertador.

\begin{tabular}{lccc}
\hline & \multicolumn{2}{c}{ Nivel de titulación } \\
Serovares de Leptospira & $\mathbf{1 / 1 0 0}$ & $\mathbf{1 / 2 0 0}$ & $\mathbf{1 / 4 0 0}$ \\
\hline L. hardjo & 3 & 6 & 1 \\
L. pomona & 2 & 3 & 0 \\
L. icterohaemorragiae & 24 & 18 & 11 \\
L. bratislava & 7 & 0 & 0 \\
\hline Total & $\mathbf{3 6}$ & $\mathbf{2 7}$ & $\mathbf{1 2}$ \\
\hline
\end{tabular}

diluciones del suero. Para el análisis de la información obtenida, utilizamos un análisis de frecuencias en MS Exel.

\section{Resultados}

La técnica de la MAT practicada a las 78 muestras demostró seropositividad en 53 de ellas, es decir que la prevalencia fue del 67.9\%. El serovar L. icterohaemorragiae fue el más predominante, seguida de la $L$. hardjo y $L$ bratislava. De las 53 personas seropositivas, 36 mostraron títulos de 1/100, 27 tenían títulos $1 / 200$ y 12 tenían títulos $1 / 400$ (Tabla 1). Con respecto a la variable sexo, de las 53 personas seropositivas $29(37.1 \%)$ eran mujeres y 24 (30.8\%) hombres (Tabla 2$)$. Con relación a la edad de los pacientes, los afectados eran personas entre los 20 y 59 años (Tabla 3).

La leptospirosis es considerada una enfermedad ocupacional. Las personas dedicadas a la agricultura han sido consideradas en alto riesgo de presentar la enfermedad, los operarios y amas de casa representaron el mayor porcentaje (73.6\%) de seropositividad (Fig. $1)$. Otros factores relacionados con la presencia de anticuerpos para Leptospira son la presencia de los ratones en las viviendas (33\%), aljibes al aire libre (29\%) y baños con aguas servidas $(25.8 \%)$ respectivamente.

\section{Discusión y Conclusiones}

La leptospirosis es una zoonosis de importancia mundial que afecta a los humanos en forma accidental, tanto en medios urbanos como rurales, en climas templados y tropicales, raramente diagnosticada, probablemente por la falta de disponibilidad de métodos de detección o por falta de conocimiento de la enfermedad. Su epidemiología está determinada por factores tales como el clima y la naturaleza de sus reservorios. Este estudio encontró una seroprevalencia del $67.9 \%$, mayor que la encontrada en estudios realizados en Colombia en zonas rurales del departamento de Cór-

Tabla 2. Seropositividad y seronegatividad a Leptospira según sexo en humanos en la zona. urbana del municipio de Puerto Libertador.

\section{Diagnóstico serológico}

Seropositivo

Seronegativo

Total

\begin{tabular}{ccccccc}
\hline Sexo & \# Muestras & $\%$ & \# Muestras & $\%$ & \# Muestras & $\%$ \\
\hline Mujer & 29 & 37.1 & 17 & 21.8 & 46 & 58.9 \\
Hombre & 24 & 30.8 & 8 & 10.3 & 32 & 41.1 \\
\hline Total & $\mathbf{5 3}$ & $\mathbf{6 7 . 9}$ & $\mathbf{2 5}$ & $\mathbf{3 2 . 1}$ & $\mathbf{7 8}$ & $\mathbf{1 0 0}$ \\
\hline
\end{tabular}


Tabla 3. Seropositividad y seronegatividad a Leptospira según edad en humanos en la zona urbana del municipio de puerto libertador.

\begin{tabular}{ccccc}
\hline & \multicolumn{4}{c}{ Diagnóstico serológico } \\
Edad (años) & \# individuos & \% & \# individuos & \% \\
\hline $20-29$ & 12 & 22.6 & 5 & 20 \\
$30-39$ & 14 & 26.4 & 9 & 36 \\
$40-49$ & 12 & 22.6 & 5 & 20 \\
$50-59$ & 13 & 24.5 & 5 & 20 \\
$60-69$ & 2 & 3.8 & 1 & 4 \\
Total & $\mathbf{5 3}$ & $\mathbf{1 0 0}$ & $\mathbf{2 5}$ & $\mathbf{1 0 0}$ \\
\hline
\end{tabular}

doba, donde se halló una prevalencia en humanos del $13.1 \%$, otros estudios realizados en operarios en el área rural andina en personas con riesgo en las explotaciones porcinas, determinaron una seroprevalencia de $22.4 \%$ y $3.9 \%$ respectivamente (Ochoa et al. 2000, Torales 2002, Uribe et al. 2003, Nájera et al. 2005).

Estos resultados difieren con los reportados a nivel mundial, que describen del 10 al $30 \%$ de seroprevalencia para Leptospira en humanos, entre los que podemos mencionar seroprevalencias de $18.9 \%$ en áreas rurales en el Estado de Yucatán, México (Zavala et al. 1984), 16\% en Baltimore, EE.UU. (Vinetz et al. 1996); $28 \%$ en Iquitos, Perú (Johnson et al. 2004) y Simões (2004) encontró en Brasil una prevalencia del $48 \%$. En un estudio epidemiológico en el área urbana y rural de Venezuela encontraron una prevalencia del $80.6 \%$ (Vado et al. 2002 ) y en Brasil encontraron en el sector rural una prevalencia del 77.2\% (De Lourdes et al. 2005), un valor muy similar al encontrado en este estudio. Este $67.9 \%$ de casos positivos a Leptospira, se debe posiblemente al resultado de la convergencia de varios factores culturales, ecológicos y socioeconómicos que hacen que la población en situación de desplazamiento del municipio de Puerto Libertador esté más expuesta a la transmisión del agente etiológico, como la extrema e inadecuada convivencia con animales domésticos y peridomésticos que son considerados como reservorios de la leptospirosis. La limitada ropa de protección los expone durante sus actividades laborales, sobre todo en el campo y en los lugares donde posiblemente los reservorios han depositado la orina contaminada con leptospiras; a esto se suma la falta de higiene con que almacenan el agua y los alimentos, que quedan expuestos a la contaminación por animales reservorio de la enfermedad. Todas estas causas se derivan del limitado nivel económico y educativo (Ochoa et al. 2000).

La alta reactividad del serovar $L$ icterohaemorragiae, no coincide con lo reportado en Colombia por Ochoa y colaboradores (2000), quienes reportan a $L$. pomona como el serovar de mayor seroprevalencia, ni con lo reportado por Uribe y colaboradores (2003), que mencionan la L. hardjo, L. canicola y L. icterohae-

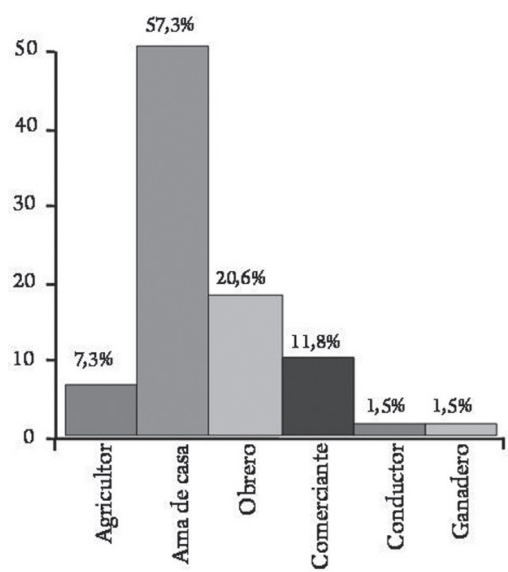

Figura 1. Prevalencia de leptospirosis asociado a la exposición ocupacional en humanos en la zona urbana del municipio de puerto libertador 
morragiae como predominantes, en ese orden. Sin embargo, nuestros resultados coinciden con algunos estudios realizados en Colombia (Ochoa et al. 2000, Vado et al. 2002, Uribe et al. 2003). Las condiciones desfavorables de vivienda, elaboradas en cartón, madera, zinc, y palmas, y la eliminación inadecuada de basuras, son factores que favorecen la presencia de roedores, siendo este el huésped del serovar L. icterohaemorragiae más predominante en las encuestas realizadas en este estudio. En menor proporción el hallazgo de L. hardjo y la L. bratislava, puede deberse a que algunas personas se dedicaban al manejo de bovinos y porcinos respectivamente (Torales 2002).

Con relación a los títulos de anticuerpos de los individuos seropositivos, 36 muestras mostraron títulos de $1 / 100$, que sugieren contacto con Leptospira, 27 individuos presentaron títulos $1 / 200$ y 12 individuos tenían títulos $1 / 400$, lo cual sugiere la presencia de signos de la enfermedad (Tabla 1); en general, estos resultados demuestran que el agente etiológico se ha mantenido constante y activo.

El MAT no puede diferenciar entre anticuerpos aglutinantes ocasionados por infección actual, reciente o pasada. Idealmente se deben analizar dos muestras consecutivas de suero para observar seroconversión o aumento de los títulos. El significado del título de una sola muestra es debatido y varía por área, por lo que diferentes títulos o puntos de corte pueden ser aplicados. Algunos consideran títulos de 1/100 positivos, otros aceptan títulos 1/200,1/400 ó 1/800 para el diagnóstico de leptospirosis actual o reciente (Wagenaar et al, 2002). , por esto para si tomamos un punto de corte de 1/400 estaríamos frente a personas que tienen leptospirosis actual o reciente. El sexo no fue una variable determinante para la presencia de esta enfermedad, coincidiendo con la investigación realizada en el departamento de Córdoba (Nájera et al. 2005) y otra hecha en México (Vado et al. 2002) y contrasta con los estudios de Cermeño-Vivas y colaboradores (2005) en Venezuela, donde se determinó una seroprevalencia en hombres que en mujeres con un $78 \%$. (Ochoa et al. 2000, O.M.S. 2001, Paparamborda 2001). Los resultados encontrados al analizar la variable edad, sugieren el hecho que la enfermedad se puede estar transmitiendo en un rango de edad más amplio que el descrito por otros autores en Colombia, Venezuela y en Cuba, donde la aparición de leptospirosis ocurre en adultos jóvenes, en la segunda y tercera década de la vida (Ochoa et al. 2000, Rodríguez 2000, Cermeño-Vivas et al. 2005Sandow \& Ramírez 2005).

La Leptospirosis al ser una enfermedad dependiente de los factores ambientales, además de la convivencia con los reservorios (v. gr. ratones, cerdos), es una situación que se convierte en un problema de salud pública que debe ser intervenido mediante programas de educación sanitaria. La Leptospirosis en humanos tiene una fuerte dependencia de la presencia de la enfermedad en los animales domésticos y peridomésticos, especialmente en las zonas urbanas, debido a la convivencia con ellos, convirtiéndose en uno de los factores de alto riesgo. El MAT aunque no es una prueba rutinaria, es muy útil para el diagnóstico de leptospirosis, especialmente en medios tropicales como el colombiano, donde circulan varios serovares de la enfermedad, pudiendo encontrarse una alta incidencia en humanos, no sólo en individuos de alto riesgo por su ocupación, sino en la población en general. En Colombia y especialmente en el departamento de Córdoba, se desconocen tanto la prevalencia como el impacto económico de la Leptospirosis; por esto recomendamos realizar investigaciones encaminadas a conocer el comportamiento de esta enfermedad. El departamento de Córdoba no cuenta con un punto de corte, por lo cual se deben adelantar estudios que incluyan seguimientos de títulos en poblaciones de alto riesgo para obtener puntos de corte en cada zona. Este estudio representa un aporte al conocimiento sobre la prevalencia de Leptospira en ambientes urbanos en el departamento, en particular en poblaciones en situación de desplazamiento. Se requieren futuros estudios sobre la transmisión y los factores de riesgo y sobre el establecimiento de un sistema de vigilancia para un mejor entendimiento de esta zoonosis ya que podría convertirse en un serio problema de salud pública.

Agradecimientos.- Agradecemos a la Universidad Nacional Abierta y a Distancia-UN$\mathrm{AD}$ por el apoyo académico, así como al Instituto Colombiano Agropecuario-ICA por la asesoría y el trabajo técnico de laboratorio y a la población del Municipio de Puerto Libertador por su colaboración. 


\section{Literatura citada}

Cermeño-Vivas, J, M Sandoval-De Mora, J Bognanno \& A Caraballo. 2005. Aspectos epidemiológicos y clínicos de la Leptospirosis en el estado Bolívar, Venezuela, 1999-2000. Comparación de LEPTO-Dipstick y antígeno termorresistente de Leptospira (TR). En: Invest. clín. 46. Maracaibo.

De Lourdes M, S Arsky, W Kleber, R Cerqueira \& AHStrugo. 2005. Probable areas of infection and ambience of occurrence of human leptospirosis in Brazil 2001-2003. Rev Cubana Med Trop, 57(1):59-60

Johnson MA, Smith H, Joeph P, Gilman RH, CT Bautista \& KJ Campos. 2004. Environmental exposure and leptospirosis, Peru. Emerg Infect Dis. 20: 1016-1022

Nájera S, N Alvis, D Babilonia, L Alvarez \& S Mattar. 2005. Leptospirosis ocupacional en una región del Caribe colombiano. Salud pública de México, 47:240-244

Ochoa JE, AR Sánchez \& I Ruiz. 2000. Epidemiología de la leptospirosis en una zona andina de producción pecuaria. Rev Panam Salud Publica 7:325-31.

O.M.S. 2001. El control de las Enfermedades Transmisibles. Organización Mundial de la Salud. Decimoséptima edición. Publicación Científica y Técnica \# 581. OPS.

Paparamborda MC. 2001. Situación de las enfermedades transmisibles en el siglo XX. Serie Monografías del Instituto de Higiene, $1: 123-132$

Rodríguez MG. 2000. Estado actual de la leptospirosis. MVZ-CORDOBA, 5: 61-63

Sandow K \& SW Ramirez. 2005. La Leptopirosis humana y bovina y su relación con los factores edafoclimaticos en una provincia de la región oriental de Cuba. Revista Electrónica de Veterinaria, 6: 1-10

Simões A. de Maria \& Lourdes, 2004. Locales probables de infección y ambiente de ocurrencia de la leptospirosis humana en el Brazil, 2001 a 2003. Memorias de II Taller Internacional y II Reunión Científica. La Habana-Cuba.

Torales, M. 2002.Leptospirosis. Carta Infectológica 2:3-6

Uribe AO, G León, AB Ríos \& PA Valencia. 2003. Leptospirosis en personas de riesgo de quince explotaciones porcinas y de la central de sacrificio de Manizales. Arch. Med. Vet, 35. (2): 205-213

Vado S, M Cárdenas, MH Laviada, PF Vargas, DB Jiménez \& VJ Zavala. 2002. Estudio de casos clínicos e incidencia de leptospirosis humana en el estado de Yucatán, México durante el período 1998 a 2000. Rev Biomedica 13:157-164

Vinetz, JM, GE Glass, CE Flexner, P Mueller \& DC Kaslow. 1996. Sporadic urban leptospirosis. Ann. Intern. Med. 125: 794-798

Wagenaar, J, RL Zuerner, D Alt \& CA Bolin. 2000. Comparison of polymerase chain reaction assays withbacteriologicculture,immunofluorescence, and nucleic acid hybridization for detection of Leptospira borgpetersenii serovar hardjo in urine of cattle. Am. J. Vet. Res, 61:316-320

Zavala, J, J Pinzón, M Flores \& AG Damian. 1984. La leptospirosis en Yucatán: Estudio serológico en humanos y animales. Salud Pública México. 26: $254-259$
Recibido: 14 de octubre de 2009 Aceptado: 10 de diciembre de 2009 